\title{
Embryonic auditory experience and maternal call recognition
}

\author{
T. JAMES REYNOLDS \\ Department of Zoology, Duke University, Durham, North Carolina 27706
}

\begin{abstract}
Peking duck embryos respond selectively to the maternal call of their species on the day before hatching, given normal embryonic auditory experience (the sounds emanating from their siblings and themselves which begin late on Day 24 of development). Groups of Peking embryos were subjected to recordings of either the mallard or the Burmese red jungle fowl maternal call beginning on Day 21 of incubation. Responses to these calls were tested on the day before hatching to determine the effects of the prenatal auditory experience. The responses of those birds exposed to the mallard maternal call were neither attenuated nor enhanced (as compared to the controls) when presented that call; those subjects which were exposed to the jungle fowl maternal call later failed to respond to that call or the mallard maternal call.
\end{abstract}

The occurrence of acoustic interactions between a mallard (Anas platyrhynchos) hen and her brood begin at the onset of peeping after the young bore into the airspace of the egg (Hess, 1972). Adult laughing gulls (Larus atricilla L.) emit a certain call with increasing frequency before the hatching of their young and the same call is used postnatally when feeding the young (Beer, 1970; Impekoven, 1970). Similarly, wood duck (Aix sponsa) hens vocalize intermittently long before (20-36 h) the exodus from the nest (Gottlieb, 1963). The suggestions offered by these investigators as to the functional significance of prenatal auditory stimulation provided by the hen are twofold: (1) the stimulation may serve to synchronize hatching, and (2) the stimulation may facilitate discrimination of speciesspecific (or even parental-specific) signals.

Substantial evidence has been previously presented supporting both proposals which are, in fact, not mutually exclusive. Synchronizing effects of embryonic auditory stimulation in some species of quail have been extensively studied by Vince (1969). Impekoven (1970) reported that prenatal experience of the adult laughing gull "crooning" call affects the neonate's postnatal response to the call. Gottlieb (1971) concluded from his research with Peking ducklings (Anas platyrhynchos) that "species-specific auditory perception is a probabilistic phenomenon"

The author expresses his gratitude to Professors P. H. Klopfer, G. J. Fischer, and D. S. Burdick for their consultatory assistance with the manuscript and/or the statistical analysis. D. Rubenstein, W. Hallahan, and C. Dewey are also due thanks, as is B. Robson for her drawing of Figure 1. This research was supported in part by NIMH Special Fellowship 51117-01 awarded to Dr. Fischer, NIMH Grant 04453 awarded to Dr. Klopfer, and a grant from the North Carolina Academy of Science awarded to the author. Address reprint requests to: Dr. Peter H. Klopfer, Department of Zoology, Duke University, Durham, North Carolina 27706. but that normally occurring prenatal stimulation is necessary for the "threshold, timing, and ultimate perfection of such perception." Tschanz (1968) provided evidence that young guillemots (Uria aalge) are able to discriminate individual parental calls postnatally if exposed to a specific parental call prenatally.

The present experiment was designed to determine the effects of prenatal auditory exposure (a conspecific maternal call or one of an alien species) on a subject's recognition of the conspecific maternal call. The issue is whether or not Peking embryos' discriminative responses to their own maternal call on the day before hatching can be effectively enhanced or attenuated as a result of the modification of their naturally occurring embryonic auditory experience.

\section{METHOD}

\begin{abstract}
Subjects
The subjects consisted of 100 Peking duck embryos, a domestic strain of the mallard, coming from 13 separate hatches. Twentyfive to 50 eggs were set per hatch group; hatchability ranged from $43 \%$ to $80 \%$ (the average number of subjects that hatched per group being 18.4). All subjects hatched $27.5 \pm 1$ days after the onset of incubation and all of those used in the experiment that were from any one group pipped within $10 \pm 2 \mathrm{~h}$ of one another.
\end{abstract}

\footnotetext{
Sound Recordings

Two recordings of maternal calls were used in this study: a call emitted by a female mallard in the fourth week of incubation and a call employed by a Burmese red jungle fowl (Gallus gallus) hen to elicit following. As compared to the mallard call, the jungle fowl maternal call was chosen on the basis of its audible disparities. The sound spectrogram of the mallard call used in this study has been published by Hess $(1972$, p. 25$)$; the spectrogram of the jungle fowl call vaguely resembles the spectrogram previously reported by Collias and Joos (1953; Figure 4, "Clucking by Broody Hen"). The duck maternal call recording consisted of six clucks emitted in $1.40 \mathrm{sec}$, the duration of each cluck being approximately $150 \mathrm{msec}$; the chicken maternal call recording con-
} 
tained five clucks emitted in $1.47 \mathrm{sec}$, the duration of each being about 110 msec.

From these recordings, four tapes were constructed and actually employed in the experiment. Two "conditioning tapes" each had one set of either the duck or the chicken maternal clucks with a period of $19.4 \mathrm{sec}$ of interim silence for a total of about $21 \mathrm{sec}$. Two "test tapes" each consisted of nine sets of one of the maternal cluck sequences with $5.9 \mathrm{sec}$ between each set for a total of about $60 \mathrm{sec}$.

\section{Testing Apparatus}

Each embryo was tested inside an Animal Acoustical Chamber, Model AC-3, manufactured by Industrial Acoustics Company, Inc. (Bronx, New York). The background noise level inside the chamber with door closed was $43 \pm 2 \mathrm{~dB}$. [All sound levels reported herein are based on measurement from Scale B (Fast) of a General Radio Co. Sound Level Meter, Type 1565-A.] Heat was supplied by an exterior heat source piped in via the chamber's ventilation system and a heat lamp placed inside the chamber for additional heat in the event that the door was opened to prepare an embryo for stimulation. Temperature was maintained at $35^{\circ} \pm 2^{\circ} \mathrm{C}$ and humidity was variable (30\%-50\%).

For recording of bill claps, two subdermal electrodes (Model E2B), manufactured by Grass Instrument Co. (Quincy, Massachusetts), were supported by clamp and ringstand and connected to a Grass Amplifier (Model 7P511).

\section{Design}

The experiment followed a 3 by 2 factorial design. The levels of the first factor were the different maternal calls (none, jungle fowl, mallard) experienced by the subjects during the $5 \pm .5$-day period in the still-air incubator, and the levels of the second factor were the two maternal calls (mallard, jungle fowl) played during a 1-min test period. The six cells generated by the model each contained three variables $\left(\overline{\mathrm{Y}}_{1}=\right.$ mean number of bill claps $/ \mathrm{min}$ exhibited in a 5 -min prestimulus period, $\bar{Y}_{2}=$ number of bill claps exhibited during the 1 -min test period, $\bar{Y}_{3}=$ mean number of bill claps/min exhibited during a 5-min poststimulus period).

\section{Procedure}

Unincubated eggs (from Ridgway Hatcheries, La Rue, Ohio) were refrigerated $5^{\circ} \pm 1^{\circ} \mathrm{C}$ for a minimum of $48 \mathrm{~h}$ before the onset of incubation to ensure uniform developmental age. All eggs were incubated for $21 \pm .5$ days in a forced-air incubator: temperature at $37.5^{\circ} \pm .5^{\circ} \mathrm{C}$, relative humidity of $65 \%-70 \%$, and background noise produced by the fans measured at the center of the incubator at $71 \mathrm{~dB}$. While in this incubator, the eggs were automatically turned $60^{\circ} 12$ times each day. After this initial incubation period, the embryos were transported to a still-air incubator housed in an isolated room supplied with background noise at $58 \mathrm{~dB}$ by an air hose. This incubator was a sound-attenuated chamber built by Hatch (1967, Appendix B), slightly modified. It consisted of an inner and an outer box (each of 1.9-cm-thick plywood) separated by a dead space about $8 \mathrm{~cm}$ wide; a heating coil was installed at the opening of the inner box in place of the inner lid. Temperature was maintained at $37^{\circ} \pm .5^{\circ} \mathrm{C}$, and the relative humidity at $65 \%-75 \%$. The eggs rested on a $29 \times 39 \mathrm{~cm}$ sheet of wire mesh elevated above a water dish which covered approximately $75 \%$ of the floor of the chamber and were manually turned $180^{\circ}$ once each day.

While in the still-air incubator, each hatch group was subjected to either no sound, the calls of the jungle fowl "conditioning tape," or the calls of the mallard "conditioning tape." The sound level for the playback of both tape loops was maintained at $71 \pm 3 \mathrm{~dB}$ measured horizontally $20 \mathrm{~cm}$ from the center of a $15-\mathrm{cm}$ speaker mounted on the side of the incubator $15 \mathrm{~cm}$ above the wire mesh (measured vertically from the center of the speaker). All subjects received the auditory stimuli from the time that they were placed in the still-air incubator until the time that they were removed for testing.
Nine $\pm 3 \mathrm{~h}$ after the onset of pipping (or, equivalently, $15 \pm 3 \mathrm{~h}$ before hatching) each subject was transported to the testing chamber, where it was prepared for recording of bill claps. A hole just large enough for easy insertion of the needle electrodes was cut in the vicinity of the pipped shell, exposing only the subject's bill. Two layers of transparent tape were then placed over the opening to stabilize the electrodes. Both electrodes were first thrust through the tape covering and were then carefully inserted into the horny covering of the subject's mandible. The recording electrode pierced the uppermost position in the center portion of the lower bill, leaving the tip protruding approximately $1-2 \mathrm{~mm}$ into the subject's mouth; the second electrode was driven into (but not through) the same bill about $2-4 \mathrm{~mm}$ to one side of the recording electrode (Figure 1). Using this procedure, a recorded bill clap can best be described as a sharp rise from baseline followed by an immediate fall below with a steady return (Figure 1). With practice, the preparation required less than $5 \mathrm{~min}$.

After the proper insertion of the electrodes, each subject was allowed to rest for $15 \mathrm{~min}$ while the operator made adjustments on the recording instrument. After this acclimation period, each subject's bill claps were recorded for $11 \mathrm{~min}$. A baseline rate was obtained for the first 5 -min interval $\left(\bar{Y}_{1}=\right.$ bill-clap scores of the prestimulus period) followed by a 1 -min stimulus period $\left(\bar{Y}_{2}\right)$ in which each subject was exposed to the calls of either the mallard or the jungle fowl "test tapes." A Uher Model 8000 recorder and a 20-cm, 8-ohm Quam speaker placed $5 \mathrm{~cm}$ from and facing the testing platform were used for the test exposure. The sound level at the opening of the egg shell was maintained at $73 \pm 2 \mathrm{~dB}$. Bill claps were recorded again in silence for the remaining 5 -min interval $\left(\overline{\mathrm{Y}}_{3}=\right.$ bill-clap scores of the poststimulus period).

Each subject was tested only once and was subsequently returned to the still-air incubator. After the final subject in a respective hatch group had been tested, all subjects in that group were transported back to the forced-air incubator to hatch. All tested subjects survived the procedure, and all tests in a single hatch group were completed before any subjects in that group completely emerged.

Of the 100 original subjects tested, data from only 58 were used in the final analysis; data were eliminated due to illegibility of any portion of a recording or accidents occurring during the test period (e.g., dislodgment of electrodes).

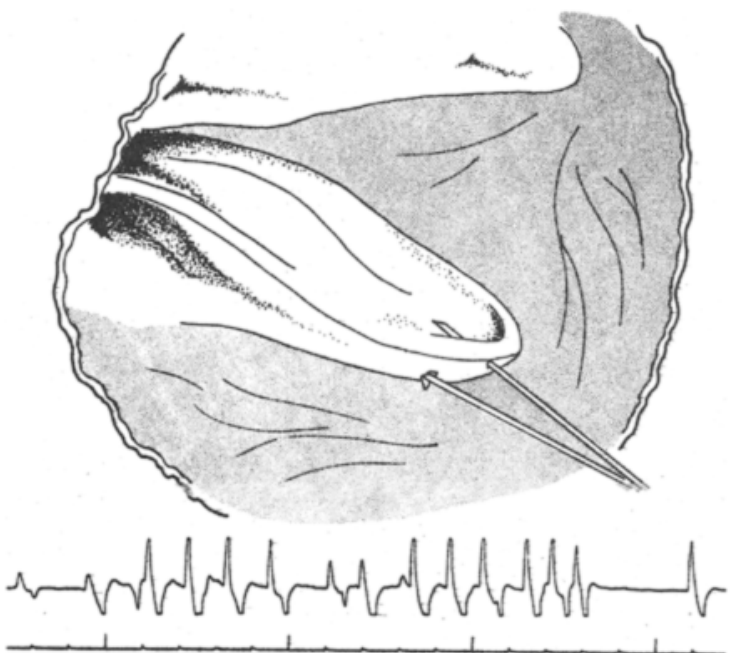

Figure 1. Above: An illustration of the preparation for recording bill claps denoting the placement of the electrodes in the lower bill. Below: A record of bill clapping with 1-sec-interval time scale beneath. 


\section{RESULTS}

Means and standard deviations of bill-clap scores for the three recording periods of each cell are given in Table 1 and means are shown in Figure 2.

As evidenced by the high standard deviations, the bill-clapping response varies considerably from subject to subject. Although no specific study was conducted to determine the maximum rate of bill clapping, subjects often exhibited rates of more than 65/ min (the average maximum score of all subjects was 64.4). The data presented in Table 1 indicate that subjects can bill-clap at rates of at least $80 / \mathrm{min}$. Hence, none of the mean scores shown in Figure 2 represent maximum rates.

Bill-clap scores exhibited within each time interval $\left(\bar{Y}_{1}, \bar{Y}_{2}, \bar{Y}_{3}\right)$ were separately subjected to a 3 by 2 (Conditioning Sound by Test Sound) factorial analysis of variance. Neither factor significantly affected the bill-clap scores exhibited in either the prestimulus $\left(\overline{\mathrm{Y}}_{1}\right)$ or poststimulus $\left(\overline{\mathrm{Y}}_{3}\right)$ periods, nor did their interaction $(\mathrm{F}<1.00, \mathrm{p}>.5$ in all cases $)$. Comparisons were also made between the scores manifested in the pre- and poststimulus periods within each cell; no significant differences were found ( $t<1.25, p\rangle .2$ in all cases). Therefore, no differences existed between the pre- and poststimulus baseline rates of activity, irrespective of the embryonic conditioning regime or the particular test stimulus.

In the factorial analysis, a significant interaction was detected for bill-clap scores in the test period $(\mathrm{F}=3.81, \mathrm{df}=2,52, \mathrm{p}=.03)$; that is, the differences of the responses exhibited during the stimulus period $\left(\overline{\mathrm{Y}}_{2}\right)$ between Cells 1 and $2\left(\overline{\mathrm{Y}}_{2_{\text {cell } 1}}-\overline{\mathrm{Y}}_{{ }_{\text {ceil } 2}}\right)$ differed significantly from the differences of the responses between Cells 3 and $4\left(\overline{\mathrm{Y}}_{2 \text { cell } 3}-\overline{\mathrm{Y}}_{2_{\text {cell }} 4}\right)$ and Cells 5 and $6\left(\bar{Y}_{2_{\text {cell } ~}}-\overline{\mathrm{Y}}_{2_{\text {cell }} 6}\right)$. When the differences of the responses of the stimulus period $\left(\overline{\mathrm{Y}}_{2}\right)$ between
Cells 1 and 2 were separately compared with the differences between Cells 3 and 4 and Cells 5 and 6 , the interaction was identified. The responses of those that experienced the jungle fowl call prenatally differed significantly from those that experienced no call $(t=2.74, \mathrm{df}=52, \mathrm{p}<.005)$; the responses of those that experienced the mallard maternal call prenatally did not differ significantly from those that experienced no call $(t=1.28, \mathrm{df}=52, \mathrm{p}>.10)$. Therefore, the responses of Peking duck embryos to the calls presented in the test period were altered only if the jungle fowl call had been presented in the embryonic environment previously.

\section{DISCUSSION}

The present findings suggest that (1) Peking embryos that experience the sounds emitted by their siblings and themselves exhibit a selective response to their own maternal call on the day before hatching, as indicated by an increase in the rate of bill clapping in response to this call when presented during the stimulus period; (2) Peking embryos' selective responses to their own maternal call on the day before hatching is neither enhanced nor attenuated by having experienced that particular call prenatally; and (3) Peking embryos that experience the jungle fowl maternal call prenatally do not respond to either their own maternal call or the jungle fowl maternal call when tested on the day before hatching.

The first result had been previously demonstrated by Gottlieb (1965). He later (1971) reported that Peking ducklings do not respond selectively to their own maternal call when pitted against a chicken maternal call if the embryos had been deprived of hearing themselves and their siblings. Recent studies (Gottlieb, 1974, 1975a, 1975b, 1975c) have dealt with this problem.

Table 1

Mean Rates and Standard Deviations of Bill-Clapping in Peking Duck Embryos

\begin{tabular}{|c|c|c|c|c|c|c|}
\hline \multirow{2}{*}{$\begin{array}{l}\text { Embryonic Auditory } \\
\text { Experience }\end{array}$} & \multicolumn{6}{|c|}{ Test Maternal Call } \\
\hline & Cell & Mallard & $\mathrm{n}$ & Cell & Jungle Fowl & $\mathrm{n}$ \\
\hline No Call & 1 & $\begin{array}{l}\bar{Y}_{1}=30.2 \pm 12.7 \\
\bar{Y}_{2}=55.2 \pm 25.7^{*} \\
\bar{Y}_{3}=31.1 \pm 13.6\end{array}$ & 8 & 2 & $\begin{array}{l}\bar{Y}_{1}=23.9 \pm 12.2 \\
\bar{Y}_{2}=13.2 \pm 6.8 \\
\bar{Y}_{3}=27.6 \pm 12.6\end{array}$ & 8 \\
\hline Jungle [owl Maternal Call & 3 & $\begin{array}{l}\bar{Y}_{1}=21.1 \pm 10.7 \\
\bar{Y}_{2}=27.7 \pm 21.8 \\
\bar{Y}_{3}=21.8 \pm 14.0\end{array}$ & 10 & 4 & $\begin{array}{l}\bar{Y}_{1}=23.4 \pm 12.4 \\
\bar{Y}_{2}=30.2 \pm 29.9 \\
\bar{Y}_{3}=27.8 \pm 13.2\end{array}$ & 11 \\
\hline Mallard Maternal Call & 5 & $\begin{array}{l}\bar{Y}_{1}=27.0 \pm 13.9 \\
\bar{Y}_{2}=47.7 \pm 32.9 * * \\
\bar{Y}_{3}=24.2 \pm 13.2\end{array}$ & 10 & 6 & $\begin{array}{l}\bar{Y}_{1}=21.7 \pm 11.8 \\
\bar{Y}_{2}=26.4 \pm 18.0 \\
\bar{Y}_{3}=28.4+18.4\end{array}$ & 11 \\
\hline
\end{tabular}

Note-Data are presented for intervals before $\left(\bar{Y}_{1}\right)$, during $\left(\bar{Y}_{2}\right)$, and after $\left(\bar{Y}_{3}\right)$ a 1-min test with either the mallard or jungle fowl maternal call. $n=$ cell size. Within each cell, the Wilcoxon matched-pairs signed-ranks test (two-tailed) was performed to evaluate departures from the prestimulus, baseline rate $\left(\bar{Y}_{1}\right)$ of activity; $p>.10$ in all but two instances.

${ }^{*} p=.02$, Wilcoxon test; ${ }^{* *} p<.01$, Wilcoxon test. 


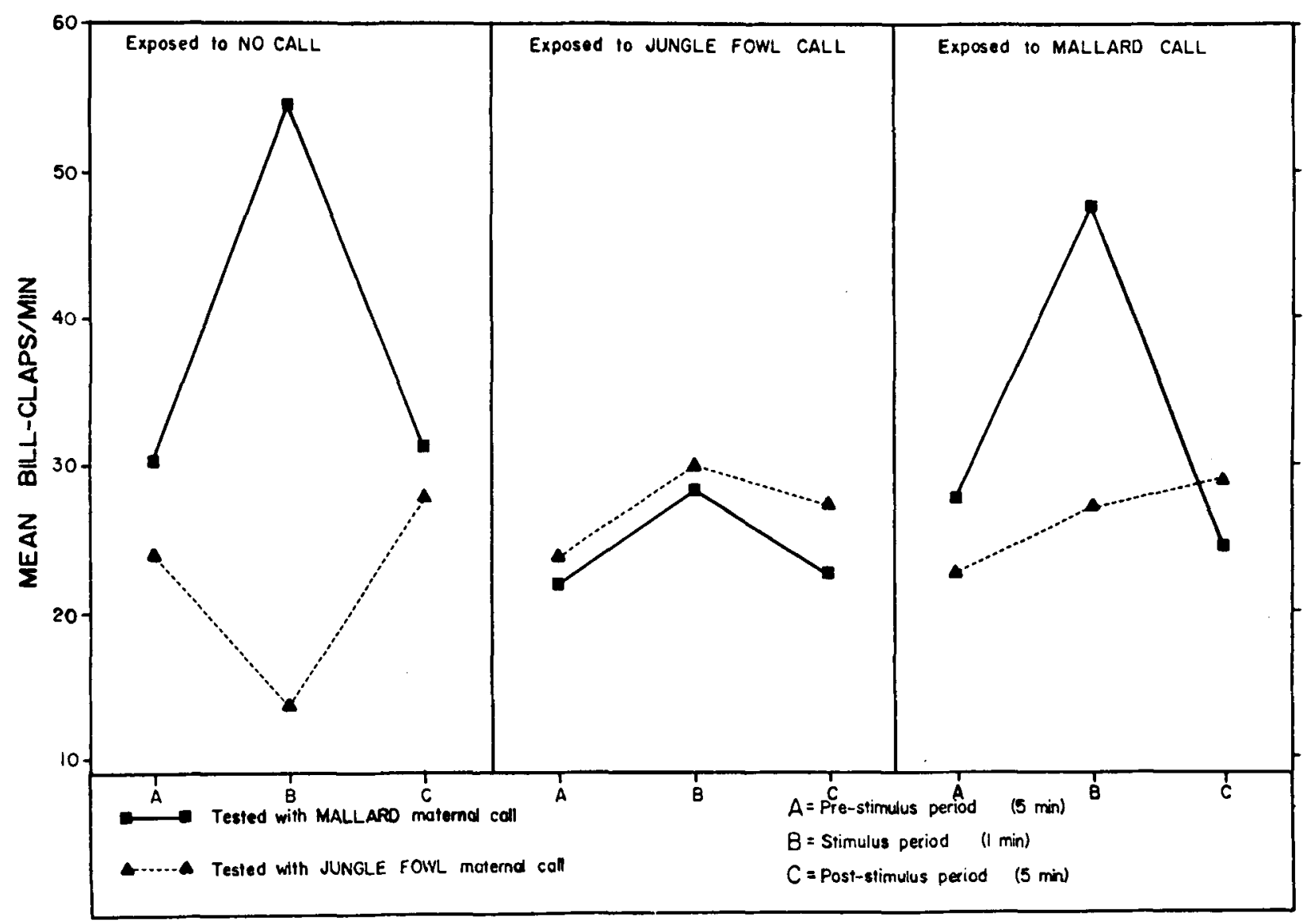

Figure 2. Mean rates of bill clapping in Peking duck embryos before, during, and after a 1-min test with either the mallard or jungle fowl maternal call. Partitioning emphasizes differing embryonic auditory environments.

The second result, a failure to quantitatively enhance the response, might have been predicted from the results of experiments conducted by Impekoven (1970). That habituation to the maternal call did not occur might also have been expected because of the obvious disadvantage this poses. However, these experiments do not exclude the possibility that other stimuli administered at different developmental ages might enhance responses: certain early postnatal auditory experiences later enhance the approach and following response of Peking ducklings to the maternal call (Gottlieb, 1966).

That the embryos exposed prenatally to the jungle fowl maternal call showed responses neither to that call nor to their own maternal call is a puzzling result. A response to both calls (a generalizing effect) is quite different from no response at all, even though one must conclude that discrimination is reduced in both cases. The experience of hearing the jungle fowl maternal call prenatally affects the subjects in such a way as to attenuate their response to their own maternal call.

The results indicate that prenatal auditory stimulation provided by a recording of a mallard hen does not contribute to the development of the correct auditory "image" or perception of the conspecific maternal call in Peking ducklings. Their exhibited ability to respond properly to the call on the day before hatching with no previous experience is not enhanced by having experienced the call earlier. Also, Peking ducklings subjected to a recording of a Burmese red jungle fowl maternal call prenatally do not respond selectively to their own maternal call on the day before hatching.

\section{REFERENCES}

BeER, C. G. On the responses of laughing gull chicks (Larus atricilla) to calls of adults. I1. Age changes and responses to different types of call. Animal Behaviour, 1970, 18, 661-667.

Collias, N., \& Joos, M. The spectrographic analysis of sound signals of the domestic fowl. Behaviour, 1953, 5, 175-188.

GoTTLIEB, G. A naturalistic study of imprinting in wood ducklings (Aix sponsa). Journal of Comparative and Physiological Psychology, 1963, 56, 89.91.

Gotrlieb, G. Prenatal auditory sensitivity in chickens and ducks. Science, 1965, 147, 1596-1598.

Gotrlieb, G. Species identification by avian neonates: Contributory effect of perinatal auditory stimulation. Animal Behaviour, $1966,14,282-290$.

GotTlueb, G. Development of species identification in birds: An inquiry into the prenatal determinants of perception. Chicago: University of Chicago Press, 1971. 
Gottlieb, G. On the acoustic basis of species identification in wood ducklings (Aix sponsa). Joumal of Comparative and Physiological Psychology, 1974, 87, 1038-1048.

GotTlres, G. Development of species identification in ducklings: 1. Nature of perceptual deficit caused by embryonic auditory deprivation. Journal of Comparative and Physiological Psychology, 1975, 89, 387-399. (a)

Gotrlieb, G. Development of species identification in ducklings: II. Experiential prevention of perceptual deficit caused by embryonic auditory deprivation. Journal of Comparative and Physiological Psychology, 1975, 89, 675-684. (b)

GotTlieb, G. Development of species identification in ducklings: III. Maturational rectification of perceptual deficit caused by auditory deprivation. Journal of Comparative and Physiological Psychology, 1975, 89, 899-912. (c)

НАтсн, J. Diversity of the song of mockingbirds (Mimus polyglottos) reared in different auditory environments. Unpublished doctoral dissertation, Duke University, 1967.
HESS, E. H. "Imprinting" in a natural laboratory. Scientific American, 1972, 227 (August), 24-31.

IMPEKOVEN, M. Prenatal experience of parental calls and pecking in the laughing gull (Larus airicilla L.). Animal Behaviour, 1970 . 19. 475-480.

Tschanz, B. Trottellummen. Die Entstehung der persönlichen Beziehungen zwischen Jungvogel und Eltern. Zeitschrift für Tierpsychologie, 1968, Suppl. 4.

VINCE, M. Embryonic communication, respiration and the synchronization of hatching. In R. A. Hinde (Ed.), Bird vocalizations. Cambridge: Cambridge University Press, 1969. Pp. 233-260.

(Received for publication June 22, 1976; revision accepted January $15,1977$. ) 